\title{
Statistical mechanics of asset markets with private information
}

\author{
Johannes Berg ${ }^{1}$, Matteo Marsili ${ }^{2}$, \\ Aldo Rustichini ${ }^{3}$, \& Riccardo Zecchina ${ }^{1}$ \\ 1 - Abdus Salam International Centre for Theoretical Physics, \\ 34100 Trieste, Italy \\ 2 - Istituto Nazionale per la Fisica della Materia (INFM), \\ Unitá Trieste-SISSA, 34014 Trieste, Italy \\ 3 - Dept. of Economics, University of Minnesota, \\ Minneapolis, MN, 55455, USA
}

August 18, 2019

\begin{abstract}
Traders in a market typically have widely different, private information on the return of an asset. The equilibrium price of the asset may reflect this information more accurately if the number of traders is large enough compared to the number of the states of the world that determine the return of the asset. We study the transition from markets where prices do not reflect the information accurately into markets where it does. In competitive markets, this transition takes place suddenly, at a critical value of the ratio between number of states and number of traders. The Nash equilibrium market behaves quite differently from a competitive market even in the limit of large economies.
\end{abstract}




\section{Introduction}

The fundamental hypothesis, which is typically adopted in financial economics, is that markets are efficient. This general hypothesis has been recently the object of a detailed, critical evaluation (see for instance [2]), nevertheless it remains an extremely useful benchmark. Many possible definitions of this hypothesis have been given. We shall adopt here the one suggested by Malkiel [6]: A market is efficient with respect to an information set if the public revelation of that information would not change the prices of the securities. Loosely speaking, this means that the information is incorporated into, or reflected by, prices.

The efficiency hypotheses may also differ for the degree, or form of efficiency. The one we are going to consider here is strong efficiency: The information set includes the information available to any of the participants in the market, including private information.

In order to address the issue of efficiency, it is fundamental to understand how precisely information scattered across different agents is aggregated into the prices of the assets that are traded in the market. Two fundamental insights of economic theory go a long way towards addressing this question.

The first insight is that the asymmetric information of different traders

may cause inefficiency of the equilibrium [1], given the strategic incentive of each agent not to reveal the information he has. The second insight is that in large markets the distortion produced by the private incentives and asymmetric information may vanish, because the benefit from the distortion, as well as the ability to distort, for any single agent may be infinitesimally small. This second insight makes the concept of market efficiency interesting and fruitful.

Here we address a specific aspect of the general question of efficiency of financial markets. How precisely does the size of the market affect efficiency? Or more precisely, how does the relative size of the market compared to the size of the uncertainty that agents are facing affect efficiency, when information available is private? The "size of the market" is an unambiguous concept, and is measured by the number of agents. The "size of the uncertainty" is less commonly used: It is defined here to be the number of possible states of nature affecting the return of the asset. We address this question in the context of a simple market, where the price of the asset is the outcome of market clearing between a total monetary demand and a fixed supply.

An alternative method, which has been frequently adopted in recent 
years, is the formulation of a precise, detailed description of the price formation process. For instance, the literature of market micro-structure analyzes the process of price formation in concrete markets, with a limited number of traders, and a well specified, usually sequential series of moves that ultimately determine the price of the asset. The emphasis is on the strategic aspects of information revelation through trade, and the role that institutional details of the market considered play in the process.

This method has provided useful insights in the working of concrete markets [5]. But as always when one adopts very specific setups, the results depend in a critical way on the details of the extensive form game. Instead, we consider here a very simple market. There is only one asset, with a return that depends on the state of nature. Traders observe an imperfect signal of the realization of the state, and can then decide how much they invest in the asset, before the price of the asset is announced. The price of the asset is then determined to clear the market. Note that the agents must decide their investment before they know the price for the period, and the price is determined to equate the total demand equal to the total amount invested, to a fixed supply. So this model is very similar to the classic model of Shapley and Shubik [8].

If agents had a complete knowledge of the state, competitive prices would equal returns in any state. This means that prices would reflect all the information on the state of nature, i.e. that the market would be efficient. When agents have only partial information, competitive prices may fail to equal returns and the market may not be efficient. It will turn out that the fundamental element for the efficiency is the size of the market compared to the size of the uncertainty. Therefore we are going to introduce as crucial parameter the relative size of the market, that is the number of agents in the market divided by the number of the states of nature. Our first main result is that the competitive equilibrium of the market becomes efficient suddenly, once a threshold in relative number of agents and events is crossed.

The second question we analyze is the size of the difference between competitive and Nash equilibrium prices. This difference, and in particular its asymptotic behavior as the number of agents tends to infinity has already been studied, although in different setups. Particularly relevant from the point of view of our research are the results that deal with economies with private information. The typical result is that the difference between Nash and competitive equilibrium vanishes as the number of agents grows. The reason for this is clear: with a large number $N$ of agents the effect of the action of each agent on the aggregate outcome becomes negligible, 
and therefore the incentive to behave differently than an agent in a competitive economy vanishes. The interesting object of study therefore is the rate of convergence to zero of this difference. This rate depends on the specific game, and concrete examples yielding different rates have been found. Two examples may be considered here as an illustration. In the Gul and Postlewaite paper [3] the rate of convergence is $\frac{1}{\sqrt{N}}$. But in Rustichini, Satterthwaite and Williams [4] the rate is much faster, and is equal to $\frac{1}{N}$.

In our model an additional feature enters the analysis: the number of agents and the size of the uncertainty, modeled by the number of states of nature, are growing together. We are going to show that the relative difference of competitive versus Nash equilibrium prices may be non vanishing in the limits of large markets. Perhaps more surprisingly, the speed of convergence depends on the amount of information available, and in a discontinuous way.

Our results are based on tools and ideas of statistical mechanics of disordered systems [9]. These same ideas have recently proven quite useful in the study of systems of heterogeneous interacting agents [10].

\section{The model}

The model is a simple market with one asset, and many traders. The return of the asset is determined in each period by the draw of a state of nature. Agents do not know the state, but observe an imperfect signal on it. After they observe the signal they have to decide the amount they invest in that period. The total amount invested, divided by the total supply of the asset, which is fixed, determines the market-clearing price. Each agent gets then the return of the asset for each unit he owns, and the market goes to the next period.

The return in each state, as well as the type of signal available to each agent, are determined once and for all before the initial period. And now we proceed to a more detailed exposition.

\section{Asset, returns, and agents}

A single asset is being traded over an infinite number of periods in a market with a set $I$ of $N$ traders. There are $N$ units of asset available. The asset has a monetary return paid at the end of each period. This return is different in each period, depends on the state of nature for that period, $\omega \in \Omega$, 
and is denoted by $R^{\omega}$. The state of nature is determined in each period, independently, according to the uniform distribution on $\Omega$, which is assumed to be a finite set円. The value of the return $R^{\omega}$ for each state $\omega$ is drawn at random before the first period, and does not change afterwards. Returns thus only change because the state of nature changes.

Traders do not observe the state directly, but have a signal on the state according to some fixed private information structure, which is determined at the initial time and remains fixed. More precisely, a signal is a function from the state $\omega \in \Omega$ to a signal space, which for simplicity we assume to be $M \equiv\{-,+\}$. The signal observed by trader $i$ if state is $\omega$ is $k_{i}^{\omega}$. The information structure available to $i$ is the vector $\left(k_{i}^{\omega}\right)_{\omega \in \Omega}$. This structure is determined, by setting $k_{i}^{\omega}=+1$ or -1 with equal probability, independently across traders $i$ and states $\omega$.

How revealing the information provided by $k_{i}$ is depends, at each state $\omega$, on the entire realization of the vector, as well as on $R^{\omega}$. If, for example $k_{i}^{\omega}=+$ for all $\omega$ such that $R^{\omega}>\bar{R}$ (and $k_{i}^{\omega}=-$ otherwise), then agent $i$ will know for sure when the return is higher than $\bar{R}$. In the random economy we consider, this is a very unlikely situation, because $R^{\omega}$ and $k_{i}^{\omega}$ are drawn independently. Still for agent $i$ the distribution of returns conditional on the signal he receives, will depend to some extent on the signal. Note that an agent who knew simultaneously the partial information of all agents would be able to know the state $\omega$, with probability one, for $N \rightarrow \infty$. Indeed the probability that there are two states $\omega$ and $\omega^{\prime}$ with different returns and that no agent can distinguish them is well approximated by $\Omega(\Omega-1) 2^{-(N+1)}$, which vanishes for $N \rightarrow \infty$, even if $\Omega$ grows proportionally to $N$ as we shall assume later.

So if the information available to each single agent happened to be revealed to all, then prices would be equal to return, for all states but a subset of states with measure tending to zero. This is what the strong efficiency hypothesis would require in our model.

\section{The market}

At the beginning of each period each trader decides to "invest" a monetary amount $z_{i}^{m}$ in the asset, depending on the signal $m=k_{i}^{\omega}$ which agent $i$ receives at that time. The total amount invested by all the agents is the demand of the asset, and the supply is fixed to $N$. The market clearing

\footnotetext{
${ }^{1}$ We shall use $\Omega$ also to denote the number of elements of $\Omega$.
} 
condition determines the price $p^{\omega}$ for the period, according to:

$$
\sum_{i \in I} \sum_{m \in M} z_{i}^{m} \delta_{k_{i}, m}=p^{\omega} N
$$

where $\delta_{i, j}$ is the Kronecker delta. When agents decide their investment $z_{i}^{m}$, they do not know the price at which they will buy the asset. Note that the price depends on the state since the amount invested by each agent depends on the state [8]. At the end of the period, each unit of asset pays a monetary amount $R^{\omega}$. If agent $i$ has invested $z_{i}^{m}$ units of money, he will hold $z_{i}^{m} / p^{\omega}$ units of asset, so his payoff will be $z_{i}^{m}\left(\frac{R^{\omega}}{p^{\omega}}-1\right)$.

The expected payoff of agent $i$ is described by a function $u_{i}$ of the entire vector of investments, as follows:

$$
u_{i}\left(z_{i}, z_{-i}\right)=\frac{1}{\Omega} \sum_{\omega \in \Omega} \sum_{m \in M} \delta_{k_{i}^{\omega}, m} z_{i}^{m}\left(\frac{R^{\omega}}{p^{\omega}}-1\right)=\sum_{m \in M} \overline{\delta_{k_{i}, m} z_{i}^{m}\left(\frac{R}{p}-1\right)},
$$

where for any real valued function $f$ defined on $\Omega$,

$$
\bar{f} \equiv \frac{1}{\Omega} \sum_{\omega \in \Omega} f^{\omega}
$$

and $z_{-i}$ denotes the strategy vector of all players but $i$. The goal of each agent $i$ is to maximize the expected value of $u_{i}$.

Agents can, if they want, invest an infinite amount in a period. For simplicity we exclude the possibility that they can sell an infinite amount, since this would complicate the market clearing condition if we insist that the price of the asset has to be positive. The results of this paper hold if we first require that investment are less than a maximum amount, and then take the limit in which this bound tends to infinity.

\section{Large markets}

We shall be interested in the behavior of the market in the limit when $N \rightarrow \infty$. For the limit to be non-trivial limit, we need to discuss how different parameters of the model behave in the limit?.

If returns are on average larger than prices, agents will tend to invest more. This in turn drives prices up towards returns. We then expect that

\footnotetext{
${ }^{2}$ We write as usual $x_{n}=O\left(y_{n}\right)$ for two sequences $\left\{x_{n}\right\}$ and $\left\{y_{n}\right\}$ if for two positive constants $c$ and $C, c \leq \frac{x_{n}}{y_{n}} \leq C$ for all $n$ sufficiently large. Also we write $\simeq$ for relations which hold almost surely as $N \rightarrow \infty$.
} 
average price $\bar{p}$ will converge to the average return $\bar{R}$ and that the fluctuations of prices with $\omega$ will adjust to those of $R^{\omega}$. The information structure however constrains the fluctuations of prices. A simple argument shows that price fluctuations are small: Note that one can write

$$
z_{i}^{m}=\frac{z_{i}^{+}+z_{i}^{-}}{2}+m \frac{z_{i}^{+}-z_{i}^{-}}{2}
$$

for $m= \pm 1$. Then for $\Omega$ large, $\overline{k_{i}} \simeq 0$ and $\overline{k_{i} k_{j}} \simeq \delta_{i, j}$ and one easily finds that

$$
\bar{p} \simeq \frac{1}{N} \sum_{i \in I} \frac{z_{i}^{+}+z_{i}^{-}}{2}
$$

and

$$
\overline{(p-\bar{p})^{2}} \simeq \frac{1}{N^{2}} \sum_{i \in I}\left(\frac{z_{i}^{-}-z_{i}^{-}}{2}\right)^{2} \propto \frac{1}{N} .
$$

A non-trivial behavior is then expected when returns have fluctuations of a comparable size. Therefore we assume that returns have the specific form:

$$
R^{\omega}=\bar{R}+\frac{\tilde{R}^{\omega}}{\sqrt{N}}
$$

where $\bar{R}>0$ and $\tilde{R}^{\omega}$ is drawn from a Gaussian distribution with zero mean and variance $\sigma^{2}$.

In particular we are interested in the transition to an efficient market, where information about returns is incorporated into prices, i.e. $p^{\omega}=R^{\omega}$. This is a set of $\Omega$ equations in $2 N$ unknown $z_{i}^{m}$. In order to allow for the possibility of observing the transition from an inefficient to an efficient market, we need to take a number of states $\Omega$ which is proportional to $N$. Hence we define $\alpha=\Omega / N$.

The key quantity we shall be interested in is the distance between vectors in $R^{\Omega}$, such as prices and returns, which is defined as usual

$$
|x-y|=\sqrt{\sum_{\omega \in \Omega}\left(x^{\omega}-y^{\omega}\right)^{2}},
$$

for all $x, y \in R^{\Omega}$.

Finally note that the parameter $\bar{R}$ can be set to 1 , without loss of generality, by a suitable choice of the units of $z_{i}^{m}$. A value $R \neq 1$ can be reintroduced at any stage of the calculation by means of dimensional analysis. This leaves us with only two control parameters $\alpha$ and $\sigma$ in the limit 
$N \rightarrow \infty$. Note now that, with our choices of parameters, the distance of returns from their average is finite: $|R-1|^{2} \simeq \alpha \sigma^{2}$.

Of course real markets have a finite, but large, number $N$ of agents and finite, but small, fluctuations of returns. As we shall see numerical simulations of finite, but large, economies are in perfect agreement with the results for $N \rightarrow \infty$.

\section{Competitive equilibrium and Nash equilibrium}

We are going to consider two equilibrium concepts for our market, competitive equilibrium and Nash equilibrium. Both give a description of what each agent does as a function of the signal observed. In the competitive equilibrium, each agent ignores the effect he has on the price. In the Nash equilibrium traders take their effect on prices into account. Here are the more precise definitions.

A competitive equilibrium is a vector $\left(z_{i}^{m}\right)_{i \in I, m \in M}$ such that:

1. for every $i$ and $m$,

$$
z_{i}^{m} \in \arg \max _{x \geq 0} \overline{\delta_{k_{i}, m}\left(\frac{R}{p}-1\right)}
$$

2. market clears at $p^{\omega}$, i.e. Eq. (1) holds.

Note that if the expected net return $\overline{\delta_{k_{i}, m}\left(\frac{R}{p}-1\right)}$ is positive then the trader invests an infinite amount when receiving signal $m$.

A Nash Equilibrium is a vector $\left(z_{i}^{m}\right)_{i \in I, m \in M}$ such that:

1. for every $i$ and $m$,

$$
z_{i}^{m} \in \arg \max _{x \geq 0} x \sum_{\omega \in \Omega} \delta_{k_{i} \omega}(m)\left(\frac{R^{\omega}}{p_{-i}^{\omega}+x / N}-1\right)
$$

where $p_{-i}^{\omega}=p^{\omega}-\sum_{m \in M} \delta_{k_{i}^{\omega}, m} z_{i}^{m} / N$ is the contribution of all other agents to the price.

2. market clears at $p^{\omega}$, i.e. Eq. (11) holds.

The difference between a competitive equilibrium and a Nash equilibrium usually vanishes as $N \rightarrow \infty$. It is easy to check this when agents have no information (for instance, when $k_{i}^{\omega}=+1, \forall i \in I, \omega \in \Omega$ ). In this case price 
cannot depend on $\omega$. The competitive equilibrium price, $p_{C}$ is equal to the expected return:

$$
p_{C}=\bar{R}
$$

while the Nash equilibrium prices are

$$
p_{N}=\left(1-\frac{1}{N}\right) \bar{R}
$$

In this case the distance in $R^{\Omega}$ between the two prices vanishes as $1 / \sqrt{N}$, for $N \rightarrow \infty$. Note that the difference $p_{C}-p_{N} \propto 1 / N$ is much smaller than fluctuations of returns, which vanish as $1 / \sqrt{N}$. We are going to see that, for a random information structure $k_{i}^{\omega}$, the difference $p_{C}^{\omega}-p_{N}^{\omega}$ is generally of order $\frac{1}{\sqrt{N}}$. In particular it is of the same order of the fluctuations in returns. We shall also see that the nature of the two states is quite different.

\section{Learning to trade}

We also consider boundedly rational agents who repeatedly trade in the market. Each agent $i$ has a propensity to invest $U_{i}^{m}(t)$ for each of the signals $m \in M$. His investment $z_{i}^{m}=\chi_{i}\left(U_{i}^{m}\right)$ at time $t$ is an increasing function of $U_{i}^{m}(t)\left(\chi_{i}: R \rightarrow R^{+}\right)$with $\chi_{i}(x) \rightarrow 0$ if $x \rightarrow-\infty$ and $\chi_{i}(x) \rightarrow \infty$ if $x \rightarrow \infty$. After each period agents update $U_{i}^{m}(t)$ according to the marginal success of the investment:

$$
U_{i}^{m}(t+1)=U_{i}^{m}(t)+\Gamma \frac{\partial u_{i}^{\omega}}{\partial z_{i}^{m}}
$$

where $u_{i}^{\omega}=\sum_{m \in M} \delta_{k_{i}^{\omega}, m} z_{i}^{m}\left(\frac{R^{\omega}}{p^{\omega}}-1\right)$ is the payoff received in state $\omega$ investing a quantity $z_{i}^{+}$or $z_{i}^{-}$depending on $k_{i}^{\omega}[7]$. As we did with the distinction between competitive equilibrium and Nash equilibrium, we distinguish between naive (or price takers) and sophisticated agents. The first do not take into account their impact on the price and hence take the partial derivative in Eq. (5) at fixed $p^{\omega}$. The latter, instead, account for the fact that if $z_{i}^{m} \rightarrow z_{i}^{m}+d z$ also the price $p^{\omega}$ changes by an infinitesimal amount and this gives a contribution to the partial derivative in Eq. (5).

\section{Results}

The study of markets' equilibria and of the asymptotic properties of the learning dynamics for $N \rightarrow \infty$, turns into the analysis of the minima of the 
Hamiltonian function

$$
H_{\eta}=|R-p|^{2}+\eta \frac{\alpha}{N} \sum_{i \in I} \frac{{z_{i}^{+2}}^{2}+z_{i}^{-2}}{2}
$$

with $\eta=0$ for competitive equilibria or naive agents, and $\eta=1$ for Nash equilibria or sophisticated agents. The first term of $H_{\eta}$ (i.e. $H_{0}$ ) is just the squared distance between prices and returns. The competitive equilibrium prices and the Nash equilibrium prices minimize the Hamiltonian. A detailed proof of this fact shall be presented elsewhere [11]. Here we offer a brief description of the main steps leading to it. We also present evidence from numerical simulations to illustrate our conclusions. First we observe that, given that $p^{\omega}-R^{\omega}=O(1 / \sqrt{N})$ is small, one can linearize the equations in $p^{\omega}$ 3. Finding equilibria becomes then a linear optimization problem which can be cast into the minimization of $H_{\eta}$.

The learning dynamics can also be linearized, leading to

$$
U_{i}^{m}(t+1)=U_{i}^{m}(t)+\Gamma \delta_{k_{i}^{\omega}, m}\left[\left(R^{\omega}-p^{\omega}\right)-\eta \frac{z_{i}^{m}}{N}\right] .
$$

Here $\eta=0$ describes price takers or naive agents whereas $\eta=1$ describes sophisticated agents. Indeed the term $z_{i}^{m} / N$ arises exactly from a derivative $\frac{\partial p^{\omega}}{\partial z_{i}^{m}}$ of price with respect to investment. The dynamics (7), in the limit $\Gamma \rightarrow 0$, turns intof a deterministic dynamics in continuum time. $H_{\eta}$ is a Lyapunov function of this dynamics which implies that the asymptotic state is described by the minima of $H_{\eta}$.

$H_{\eta}$ is a non-negative definite quadratic form of the dynamical variables $\left(z_{i}^{m}\right)_{i \in I, m \in M}$. This means that there is a single minimum (which is either a single point or a connected set).

In order to study the properties of the minima of $H_{\eta}$, we resort to tools of statistical mechanics. $H_{\eta}$ depends on the particular realization of the of two random variables: the random information structure $k_{i}^{\omega}$ and of the returns $R^{\omega}$. In the language of statistical mechanics, these two random variables represent the disorder in our system. However, in the limit $N \rightarrow \infty$, the

\footnotetext{
${ }^{3}$ E.g. $R^{\omega} / p^{\omega}-1 \simeq\left(R^{\omega}-p^{\omega}\right) / \bar{R}$, where again $\simeq$ means that we are neglecting terms which vanish at least as $N^{-1 / 2}$ relative to the one retained.

${ }^{4}$ The idea is to consider a time interval $\Delta t=d \tau / \Gamma$ where $d \tau$ is small but much larger than $\Gamma$. Then $U_{i}^{m}(t+\Delta t)-U_{i}^{m}(t)$ can be estimated to linear order in $d \tau$ using the law of large numbers for $\Delta t \rightarrow \infty$ as $\Gamma \rightarrow 0$.
} 


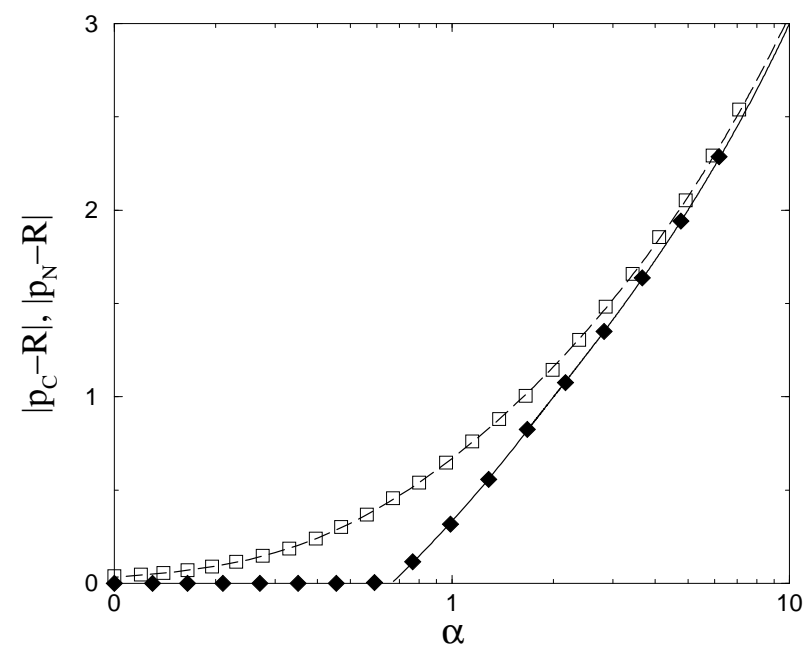

Figure 1: Distance $|p-R|=\sqrt{\sum_{\omega}\left(p^{\omega}-R^{\omega}\right)^{2}}$ of prices from returns in the competitive (full line and diamonds) and Nash equilibria (dashed line and squares). Full lines and dashed lines present the analytical graphs; diamonds and squares trace the numerical simulations of systems with $N=200$ agents and small learning rates. Averages are taken over 100 realizations of the disorder in the stationary state.

statistical behavior is independent of the specific realization of the disorder 9 . Averages over the disorder are handled by the replica method of statistical mechanics [9], which is briefly discussed in the appendix. A similar analysis for a system of interacting heterogeneous adaptive agents has been carried out, with the same techniques, in Ref. [10]. Below we discuss the results in the two cases of competitive or Nash equilibria as a function of the two parameters $\alpha$ and $\sigma$.

\section{Equilibria and the transition to efficient markets}

In the competitive equilibrium agents minimize the distance between prices and returns. As the number of agents increases, i.e. as $\alpha=\Omega / N$ decreases, agents are collectively more efficient in driving prices close to returns. Indeed

\footnotetext{
${ }^{5}$ This statement applies to some of the observables, which are called self-averaging. Typically the average value $\sum_{i} O_{i} / N$ of a quantity $O_{i}$ defined for each agent $i$ is selfaveraging (i.e. attain a.s. a fixed value) by virtue of the law of large numbers when $N \rightarrow \infty$. We refer to ref. 9] for further discussion.
} 


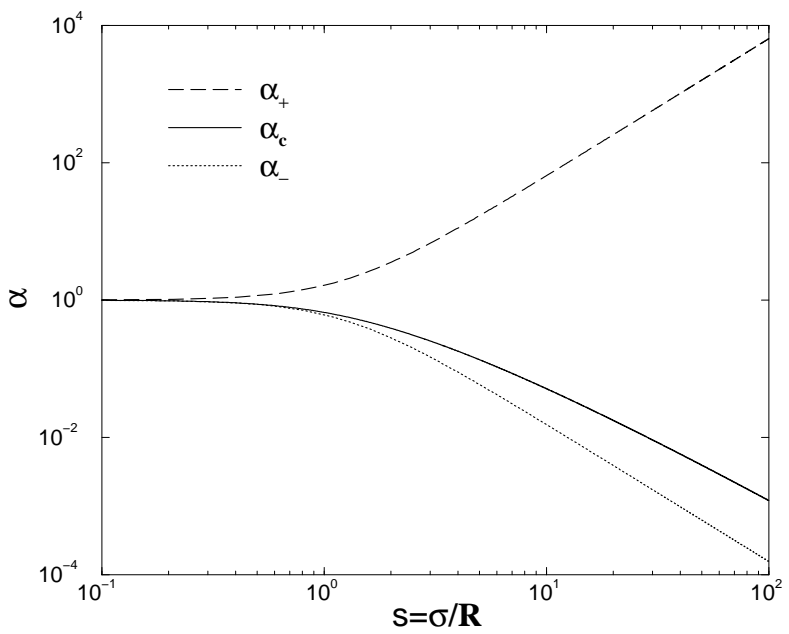

Figure 2: Phase diagram for competitive markets in the $(s, \alpha)$ plane. The curve $\alpha_{c}(s)$ (full line) separates the efficient $\left(\alpha<\alpha_{c}\right)$ from the inefficient $\left(\alpha>\alpha_{c}\right)$ phase. For $\alpha_{-}<\alpha<\alpha_{+}$agents invest at most once, whereas for $\alpha>\alpha_{+}$or $\alpha<\alpha_{-}$agents invest a positive amount under both signals.

the distance $|R-p|$ decreases as $\alpha$ decreases, as shown in Fig. 1. The distance vanishes at a critical point $\alpha_{c}$ which marks a second order phase transition, in the statistical mechanics approach. The value of $\alpha_{c}$ depends on the intensity $\sigma$ of fluctuations of returns, as shown in Fig. 2. The region $\alpha<\alpha_{c}$ is characterized by the condition $H_{0}=0$, which means $p^{\omega}=R^{\omega}$ for all $\omega \in \Omega$. This means that the market efficiently aggregates the information dispersed across agents into the price. The efficient phase, where $H_{0}=0$, shrinks as $\sigma$ increases. This is reasonable because as the fluctuations in $R^{\omega}$ increase, it becomes harder and harder for the agents to incorporate them into prices.

In order to analyze how agents behave, as a function of $\alpha$ and $\sigma$, it is useful to introduce the quantity

$$
q_{1}=\frac{1}{N} \sum_{i=1}^{N}\left(\frac{z_{i}^{+}-z_{i}^{-}}{2}\right)^{2} .
$$

This measures how differently agents behave upon receiving the two different signals, i.e. how much they use the signal they receive. This is plotted in figure 3 as a function of $\alpha$ for $\sigma=1$. First we observe that $q_{1}$ increases as 


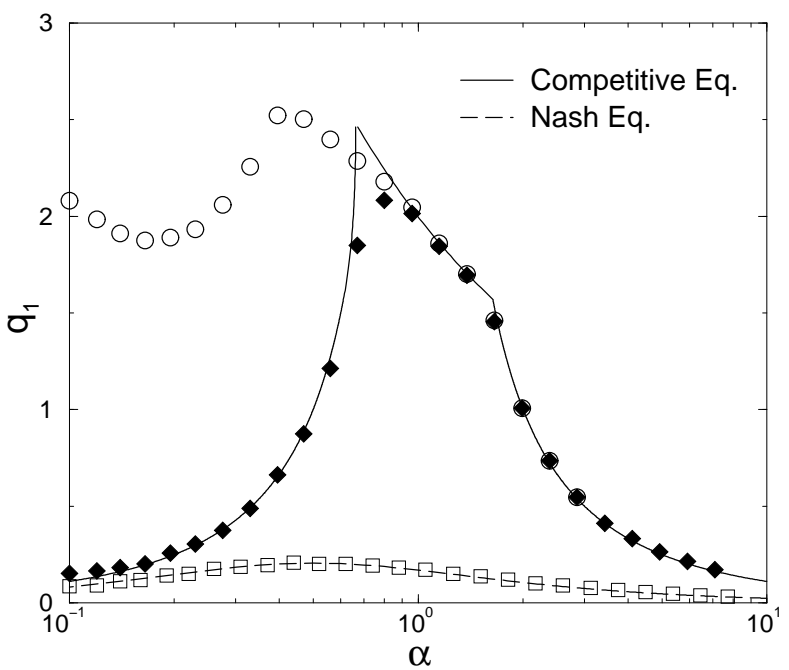

Figure 3: Parameter $q_{1}$ in the competitive (full line, diamonds and circles) and Nash equilibria (dashed line and squares). Lines present the graphs of the analytical solution; diamonds and squares refer to numerical simulations of systems with $N=200$ agents and small learning rates. Averages are taken over 100 realizations of disorder in the stationary state. The two sets of simulations, in the competitive equilibria, refer to different initial conditions: symmetric (diamonds) and asymmetric (circles).

$\alpha_{c}$ is approached both from above or below, and that it displays a cusp at $\alpha_{c}$. A second singularity appears at a larger value of $\alpha$ - called $\alpha_{+}-$whose behavior as a function of $\sigma$ is also shown in Fig. 2. In the region $\left[\alpha_{c}, \alpha_{+}\right)$ agents invest a non-zero amount at most for one of the two signals they receive. Actually in this region there is also a fraction $\phi$ of agents who do not invest $\left(z_{i}^{+}=z_{i}^{-}=0\right)$ at all. This fraction increases as $\alpha_{c}$ is approached from above, suggesting that it becomes harder and harder for agents to find profitable opportunities with $R^{\omega}>p^{\omega}$, as $\alpha \rightarrow \alpha_{c}$.

For $\alpha>\alpha_{+}$, instead, all agents make positive investments under both signals $\left(z_{i}^{+}>0\right.$ and $\left.z_{i}^{-}>0\right)$. This sudden change manifests itself in the singular behavior of $q_{1}$ at $\alpha_{+}$. It is remarkable that a mixed state, with some agents investing all the time and some others investing under one signal at most, is not possible. As $\alpha$ increases, for $\alpha>\alpha_{c}$, the information complexity increases and the market becomes more and more inefficient. Prices follow returns to some extent, in the sense that when $R^{\omega}$ is larger than the average 
$-\bar{R}$, the price $p^{\omega}$ is also larger than $\bar{p}$. But the slope of a regression of $p^{\omega}-\bar{p}$ versus $R^{\omega}-\bar{R}$ is less than one and it vanishes as $\alpha^{-1}$ for $\alpha \rightarrow \infty$.

As the complexity of fluctuations of returns increases, increasing $\alpha$, the partial information of agents becomes less and less useful. Hence for $\alpha \rightarrow \infty$ one recovers the case without information, where $p^{\omega}=\bar{R}$, and $H_{0} \rightarrow \alpha \sigma^{2}$ converges to the fluctuations of returns themselves.

From the agent's point of view the equilibrium in the efficient phase $\left(\alpha<\alpha_{c}\right)$ is not unique. This means that adaptive agents following the above learning dynamics will end up in a states $\left\{z_{i}^{m}\right\}$ which depends on the initial conditions $\left\{U_{i}^{m}(t=0)\right\}$ (prices, of course, do not depend on the initial condition, because $p^{\omega}=R^{\omega}$ for all $\omega \in \Omega$ ). This feature is captured by the parameter

$$
q_{0}=\frac{1}{N} \sum_{i=1}^{N} \frac{z_{i}^{+}-z_{i}^{-}}{2} \frac{z_{i}^{+}-z_{i}^{\prime-}}{2}
$$

in the statistical mechanics approach, where $z_{i}^{m}$ and $z_{i}^{\prime m}$ represent two different systems of agents, with different initial conditions. We find that $q_{0} \neq q_{1}$, in general, for $\alpha<\alpha_{c}$. Fig. 3 reports the value of $q_{1}$ for symmetric initial conditions $U_{i}^{m}(0)=0$ for all $i \in I$ and $m \in M$. This matches the results of numerical simulations with those initial conditions but Fig. 3 also shows that asymmetric initial conditions $\left(U_{i}^{m}(0)=10 m\right.$ for all $i \in I$ and $\left.m= \pm 1\right)$ yield a different value of $q_{1}$ and hence a different equilibrium.

\section{Market impact and Nash equilibria}

The fact that the market impact $\frac{\partial p^{\omega}}{\partial z_{i}^{m}}$ of each agent is of order $1 / N$ might suggest that prices in the Nash equilibrium should be close to those in the competitive equilibrium. More precisely one might expect that for every $\omega, p_{C}^{\omega}-p_{N}^{\omega}=O(1 / N)$ as in the case where agents have no information on the state of nature that we have discussed above. For large $N$ this is much smaller than the distance between prices and returns because $p_{C}^{\omega}-R^{\omega}=$ $O(1 / \sqrt{N})$ in the competitive equilibrium. This suggests that Nash equilibria behaves similarly to competitive equilibria, and that the distinction vanishes as $N \rightarrow \infty$.

This conclusion however is not correct when agents have private information, as one can read from Eq. (6). Indeed, the term proportional to $\eta$ in Eq. (5), at the competitive equilibrium is of the same order in $N$ of the first term. 
In fact

$$
\left|p_{C}-p_{N}\right| \geq\left|p_{C}-R\right|-\left|R-p_{N}\right| \geq 0
$$

by the reverse triangle inequality $\left(\left|p_{C}-R\right|-\left|R-p_{N}\right| \geq 0\right.$ holds because $p_{C}^{\omega}$ is the feasible price closest to $R^{\omega}$ ). Fig. 11 shows that the right hand side is finite. This contrast with the case of no information where $\left|p_{c}-p_{N}\right| \propto$ $1 / \sqrt{N} \rightarrow 0$ as $N \rightarrow \infty$. This case is only recovered in the limit $\alpha \rightarrow \infty$, in which $\left|p_{C}-R\right|-\left|R-p_{N}\right| \rightarrow 0$ (see Fig. 1).

The second term of Eq. (6) dramatically changes the statistical behavior of the market: i) the phase transition disappears: the distance between prices $p_{N}^{\omega}$ and returns $R^{\omega}$ smoothly decreases, as shown in figure 1, and it vanishes as $\alpha \rightarrow 0$. ii) the equilibrium is unique in both prices and investment for all $\alpha>0$. The asymptotic behavior of learning dynamics does not depend on initial conditions. iii) Agents always invest at least under one signal, for all $\alpha>0$. All agents who invest under both signals invest, on average over the two signals, the same quantity? iv) average price $\bar{p}$ is lower than average return $\bar{R}$, by a term of order $1 / N$. As a consequence, the payoff of agents is not zero, as in competitive equilibria, but rather it is proportional to $1 / N$.

Again as $\alpha \rightarrow \infty$ the fluctuations of prices with $\omega$ die out. This explains why, in that limit the price converges to the one without information. At the same time, as $\alpha \rightarrow \infty\left|p_{N}-R\right|-\left|p_{C}-R\right| \rightarrow 0$ and Nash prices converge to competitive prices (see Fig. 四).

\section{Learning rates and price volatility}

Let us explore the dependence of the results discussed so far on the learning rate $\Gamma$. In the limit $\Gamma \rightarrow 0$ the dynamics is deterministic and hence prices converge to equilibrium prices and the investment of agents also converges to a fixed point equilibrium. When $\Gamma>0$ the dynamics becomes stochastic and fluctuations occur. These do not affect average prices $\langle p \mid \omega\rangle$, which attain their $\Gamma \rightarrow 0$ value?. On the contrary, price volatility increases, as

\footnotetext{
${ }^{6}$ This is easily seen from the equilibrium condition, which requires $\overline{\delta_{k_{i}, m}(R-p)}-$ $\overline{\delta_{k_{i}, m}} \frac{z_{i}^{m}}{N} \leq 0$ with the equality holding whenever $z_{i}^{m}>0$. Taking the sum on $m$, we find $\overline{z_{i}^{k_{i}}} / N=\overline{(R-p)}$ for all agents who invest under both signals. The same conditions imply that there cannot be an agent who never invests. Indeed if $z_{i}^{m}=0$, it must be that $\overline{\delta_{k_{i}, m}(R-p)} \leq 0$. The sum on $m$ gives $\overline{(R-p)} \leq 0$ which cannot be correct: agents will never spend more that they get at equilibrium.

${ }^{7}$ The notation $\langle\ldots\rangle$ is intended here for long time averages in the stationary state of the dynamics. The symbol $\langle\ldots \mid \omega\rangle$ stands averages conditional on the state $\omega$.
} 


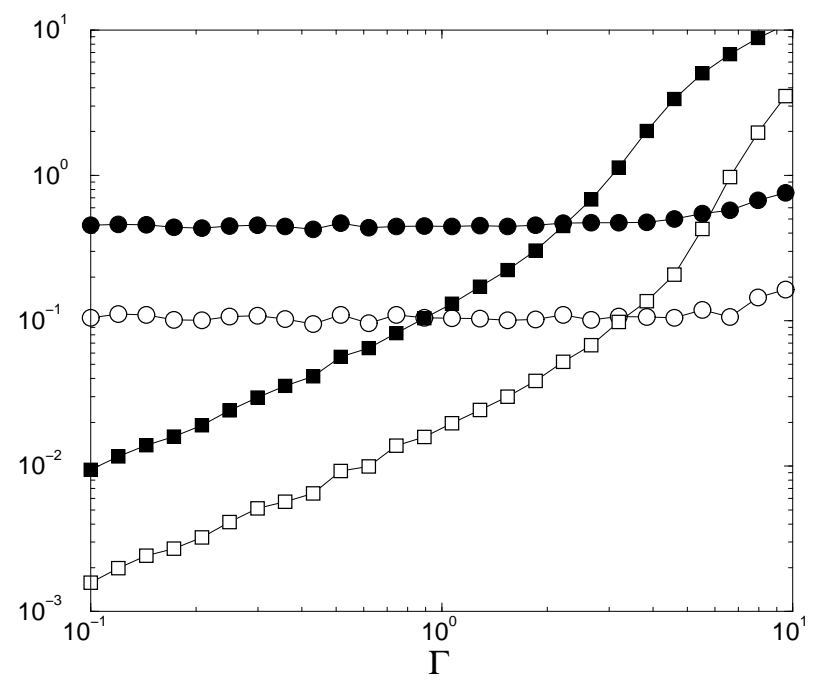

Figure 4: Squared distance $|R-\langle p\rangle|^{2}$ of prices from returns for $\alpha=s=1$ as a function of $\Gamma$ (circles). Price fluctuation $\delta p^{2}$ are also plotted (squares). Open symbols refer to Competitive equilibria whereas full symbols refer to Nash equilibria.

shown in Fig. 4. For both Competitive and Nash equilibria we find that the distance of average prices to returns $|R-\langle p\rangle|$ stays remarkably constant when $\Gamma$ increases over two decades. On the contrary, price fluctuations $\delta p^{2} \equiv \sum_{\omega}\left\langle(p-\langle p \mid \omega\rangle)^{2} \mid \omega\right\rangle / \Omega$ increase approximately linearly with $\Gamma$ in the same range.

Hence excess volatility arises in these model markets when agents react too fast or strongly to price adjustments.

\section{Conclusions}

We have characterized the relative size of the market that gives at equilibrium market efficiency. The economy we have considered is very simple: there is only one asset. In addition, to simplify the analysis, we have considered the case of agents with unbounded wealth.

Our results generalize in a straightforward way to more general setups. This is true in particular for the economies with many assets. As long as agents have no budget constraint, they can invest independently in each asset 
separately. In other words agents do not introduce additional correlation in the prices of different assets beyond those already present in the returns.

\section{A Appendix: the statistical mechanics analysis}

In order to study the minima of $H_{\eta}$, we introduce the partition function

$$
Z(\beta)=\int_{0}^{\infty} d z_{1}^{+} \int_{0}^{\infty} d z_{1}^{-} \ldots \int_{0}^{\infty} d z_{N}^{+} \int_{0}^{\infty} d z_{N}^{-} e^{-\beta H_{\eta}\left\{z_{i}^{m}\right\}}
$$

The integrals, in the limit $\beta \rightarrow \infty$ are dominated by the configurations $\left\{z_{i}^{m}\right\}$ for which $H_{\eta}$ is minimal. The central quantity to compute is the free energy $f_{\beta}=-\beta^{-1} \log Z(\beta)$. This has to be averaged over the realization of the disorder $\left\{k_{i}^{\omega}, R^{\omega}\right\}$. One can reduce the problem of computing the average $\langle\log Z(\beta)\rangle$ of the logarithm to that of computing averages of moments $\left\langle Z^{n}(\beta)\right\rangle$ by the identity $\log Z=\lim _{n \rightarrow 0}\left(Z^{n}-1\right) / n$. This is known as the replica trick [9] because, for integer $n, Z^{n}(\beta)$ is the partition function of $n$ non-interacting replicas $\left\{z_{i, 1}^{m}\right\}, \ldots,\left\{z_{i, n}^{m}\right\}$ of the system, with the same realization of disorder. Taking the average over the disorder introduces an effective interaction between replicas. The resulting expression is handled in such a way as to be able to use saddle point methods to compute it in the limit $N, \beta \rightarrow \infty$. An assumption on the symmetry of the minima with respect to permutation of replicas is necessary at this point. This symmetry can be broken in case of multiple minima, because different replicas may endup in different minima. This is not our case because $H_{\eta}$ is a non-negative definite quadratic form. So the solution in our case is replica symmetric (see

[9] for more details). A more detailed discussion of these steps in the context of a model of adaptive heterogeneous agents is given in Ref. [10].

The final result is that we can write

$$
E_{k, R}\left[\min _{\left\{z_{i}^{m}\right\}} H_{\eta}\right]=\lim _{\beta \rightarrow \infty} \min _{q_{0}, \hat{q}_{0}, x, w, \hat{R}} f_{\beta}\left(q_{0}, \hat{q}_{0}, x, w, \hat{R}\right)
$$

where

$$
\begin{aligned}
f_{\beta}\left(q_{0}, \hat{q}_{0}, x, w, \hat{R}\right)= & \frac{1}{\beta} \ln (1+x)+\frac{q_{0}+\sigma^{2}}{1+x}+\frac{x \hat{q}_{0}}{\alpha}-\frac{w q_{0}}{\alpha}+\frac{2 \hat{R}}{\alpha} \\
& -\frac{2}{\alpha \beta} \int_{-\infty}^{\infty} \frac{d t e^{-t^{2} / 2}}{\sqrt{2 \pi}} \ln \int_{z \geq 0} d^{2} z e^{-\beta V_{t}(z)}
\end{aligned}
$$


and, with $z=(\bar{z}+\Delta, \bar{z}-\Delta)$

$$
V_{t}(z)=\frac{w+\alpha \eta}{2} \Delta^{2}-\sqrt{\hat{q}_{0}} t \Delta+\frac{\alpha \eta}{2} \bar{z}^{2}-\hat{R} \bar{z} .
$$

Here $x=\beta\left(q_{1}-q_{0}\right)$ is a combination of the two order parameters whereas $\hat{q}_{0}, w$ and $\hat{R}$ arise as auxiliary variables (Lagrange multipliers).

The minimization problem contains the statistical information on the Gibbs probability distribution $e^{-\beta H_{\eta}}$ over the space spanned by $\left\{z_{i}^{m}\right\}$, averaged over all realizations of disorder. We refer the reader to ref. [9] for a deeper discussion. Let it suffice to say that this probability distribution factorizes over agents, which is typical of systems with mean field interaction. Hence the probability that an agent invests fractions $z \in A$ where $A$ is any subset of $R_{+}^{2}$, is given by

$$
\operatorname{Prob}\{z \in A\}=\int_{A} d z \int_{-\infty}^{\infty} \frac{d t e^{-t^{2} / 2}}{\sqrt{2 \pi}} \frac{e^{-\beta V_{t}\left(z^{+}, z^{-}\right)}}{\int_{\zeta \geq 0} d^{2} \zeta e^{-\beta V_{t}(\zeta)}}
$$

where the parameters of $V_{t}$ are those which satisfy the five first order conditions $\frac{\partial f_{\beta}}{\partial q_{0}}=0, \frac{\partial f_{\beta}}{\partial \hat{q}_{0}}=0, \frac{\partial f_{\beta}}{\partial x}=0, \frac{\partial f_{\beta}}{\partial w}=0$ and $\frac{\partial f_{\beta}}{\partial \hat{R}}=0$.

As $\beta \rightarrow \infty$, the integrals in Eq. (12) are dominated by the minimum $z^{*}$ of $V_{t}(z)$ in $R^{2}$. The calculation leading to the final result are lengthy. We just report the final result.

\section{A.1 The case of $\eta=0$}

When $\eta=0$ a degeneracy of solutions occurs for $\alpha<\alpha_{c}$. In other words, $H_{0}$ does not attain its minimum at a single isolated point, but rather on a connected set of points. Each point is the equilibrium of a market with some initial condition. As one varies the initial conditions, the equilibrium moves on the set.

The statistical mechanics approach takes an uniform average over all the points of this set. The results derived in this way, do not describe any particular market equilibrium, but rather a uniform distribution of equilibria resulting from some distribution of initial conditions. Since the mapping between initial conditions and equilibria is not known, it is not clear what exactly the statistical mechanics approach describes.

There is way out, in these kind of situations, which is to introduce a term in the functional to be minimized which breaks the degeneracy and selects one point in the set. In the limit when the strength of this term 
vanishes, one recovers the statistical properties of the original system in the selected equilibrium. The $\eta$ term is exactly of this form. Furthermore it is a perturbation which preserves the symmetries of the system $\left(k_{i} \rightarrow-k_{i}\right.$ for some $i$ ). With $\eta=0^{+}$we then expect to find the properties of markets with symmetric initial conditions $\left(U_{i}^{+}(0)=U_{i}^{-}(0)\right)$.

The solution for $\eta=0^{+}$takes a parametric form. Let us define the functions

$$
\begin{aligned}
& \psi_{r}(\tau)=\sqrt{\frac{2}{\pi}} e^{-\tau^{2} / 2}-\tau \operatorname{erfc}(\tau / \sqrt{2}) \\
& \psi_{q}(\tau)=\left(1+\tau^{2}\right) \operatorname{erfc}(\tau / \sqrt{2})-\sqrt{\frac{2}{\pi}} \tau e^{-\tau^{2} / 2} \\
& \psi_{x}(\tau)=\operatorname{erfc}(\tau / \sqrt{2}) .
\end{aligned}
$$

For $\tau \in\left[0, \tau_{c}\right]$, where $\tau_{c}$ is the solution of $\psi_{q}(\tau)+\sigma^{2} \psi_{r}(\tau)=\psi_{x}(\tau)$ we have

$$
\begin{aligned}
\alpha & =\psi_{q}(\tau)+\sigma^{2} \psi_{r}(\tau) \\
q_{0} & =\frac{\psi_{q}(\tau)}{\psi_{r}^{2}(\tau)} \\
|R-p| & =\frac{\psi_{q}(\tau)+\sigma^{2} \psi_{r}(\tau)-\psi_{x}(\tau)}{\psi_{r}(\tau)}
\end{aligned}
$$

which holds in the interval $\alpha \in\left[\alpha_{c}, 1+\frac{2}{\pi} \sigma^{2}\right]$, where $\alpha_{c}=\alpha\left(\tau_{c}\right)$. Notice that $H \rightarrow 0$ as $\alpha \rightarrow \alpha_{c}$.

For $\alpha \in\left[\left(1+\frac{2}{\pi} \sigma^{2}\right)^{-1}, \alpha_{c}\right]$ we find

$$
\begin{aligned}
\alpha & =\frac{\psi_{x}(\tau)}{\psi_{q}(\tau)+\sigma^{2} \psi_{r}(\tau)} \\
q_{0} & =\frac{\psi_{q}(\tau)}{\psi_{r}^{2}(\tau)} \\
|R-p| & =0
\end{aligned}
$$

again for $\tau \in\left[0, \tau_{c}\right]$.

In these two regions, a fraction $\phi=\operatorname{erf}(\tau / \sqrt{2})$ of agents never invests, whereas the others invest only under at most one signal. The fraction $\phi$ is largest at the critical point $\alpha_{c}$, i.e. for $\tau=\tau_{c}$.

Outside this region we find an explicit solution: For $\alpha \geq 1+\frac{2}{\pi} \sigma^{2}$, we find $q_{0}=1 / \alpha$ and $|R-p|=(1-1 / \alpha) \sqrt{1+\alpha \sigma^{2}}$. Hence the bare fluctuations 
of returns $|R-1|=\sqrt{\alpha \sigma^{2}}$ are recovered in this limit. In the region $\alpha \leq$ $1 /\left(1+\frac{2}{\pi} \sigma^{2}\right)$ we find $q_{0}=\alpha$ and $|R-p|=0$. Agents invest under both signals and the distribution of their average investment $\left(z^{+}+z^{-}\right) / 2$ is exponential.

\section{A.2 The case of $\eta=1$}

The study of the minimum of the potential $V_{t}(z)$ is quite different in this case. One has to distinguish two regions of integration of $t$, according to whether the minimum of $V_{t}(z)$ belongs to $R_{+}^{2}$ or not. The first case describes agents who invest under both signals, and our solution confirms that they all invest on average the same amount. The second case describes the fraction of agents who only invest once.

The solution again depends parametrically on $\tau$ and on a parameter $y \in[1,2]$ which satisfies the equation:

$$
\begin{aligned}
1-\frac{I_{0}(\tau)}{1+y}=\frac{(2-y)(y-1)}{y}\{1 & -\frac{I_{0}(\tau)+\left[I_{1}(\tau)-\tau\right] \tau+2 I_{0}(\tau) y}{(1+y)^{2}} \\
& \left.+\frac{\sigma^{2}\left[\tau+\left(I_{1}(\tau)+\tau-I_{0}(\tau) \tau\right) y\right]^{2}}{(1+y)^{2}}\right\}
\end{aligned}
$$

with $I_{0}(\tau)=\operatorname{erfc}(\tau / \sqrt{2})$ and $I_{1}(\tau)=\sqrt{2 / \pi} e^{-\tau^{2} / 2}$. We have

$$
\begin{aligned}
q_{0} & =\frac{(1+y)^{2}+\tau^{2}-(1+2 y) I_{0}(\tau)-\tau I_{1}(\tau)}{\left[\tau(1+y)-y \tau I_{0}(\tau)+y I_{1}(\tau)\right]} \\
\alpha & =\left(q_{0}+\sigma^{2}\right)\left\{\frac{(y-1)\left[\tau(1+y)-y \tau I_{0}(\tau)+y I_{1}(\tau)\right]}{y(1+y)}\right\}^{2} \\
|R-p| & =\left(q_{0}+\sigma^{2}\right)(y-1)^{2}\left[\frac{\tau}{y}-\frac{\tau I_{0}(\tau)-I_{1}(\tau)}{1+y}\right]
\end{aligned}
$$

This has two solutions $y^{ \pm}$for $\tau>\tau_{0}$, which describe the low and high $\alpha$ regions. There is no singularity or discontinuity in the solution when $\tau \rightarrow \tau_{0}$ (corresponding to $\alpha \approx 0.502 \ldots$ for $\sigma^{2}=1$ ). The fraction of agents playing only once is given by $I_{0}(\tau)$. 


\section{References}

[1] A first influential paper to prove this statement in a formal model is Akerlof, G., (1970), "The Market for Lemons", The Quarterly Journal of Economics, 89, 488-500.

[2] Shiller, R. S., Market Volatility, MIT Press, Cambridge MA, (1989), or Lo, A. W. and Mackinlay, A. C., A Non-Random Walk Down Wall Street, Princeton University Press, (1999).

[3] Gul, F. and Postlewaite, A., (1992), "Asymptotic Efficiency in Large Exchange Economies with Asymmetric Information", Econometrica, 60, 6, 1273-1292.

[4] Rustichini, A., Satterthwaite, M. and Williams, S., (1994), "Convergence to Efficiency in a Simple market with Incomplete Information", Econometrica, 62, 5, 1041-1063.

[5] A very useful survey of the results in this area is in Maureen O'Hara, (1994), Market Microstructure Theory, Blackwell, Malden, MA.

[6] Malkiel, B., (1992), "Efficient Market Hypothesis", in Newman, P., Milgate, M., and Eatwell, J., (eds.) New Palgrave Dictionary of Money and Finance, MacMillan, London. A classic paper for the efficient market hypothesis is Fama, E. F., "Efficient Capital Markets: A Review of Theory and Empirical Work", Journal of Finance, 25, 383-420, (1970). For recent and critical evaluations see for instance Shiller, R. S., Market Volatility, MIT Press, Cambridge MA, (1989), or Lo, A. W. and Mackinlay, A. C., A Non-Random Walk Down Wall Street, Princeton University Press, (1999).

[7] The properties of this learning mechanism, well known among psychologists, have been recently widely discussed in the economics literature. See for instance Borgers T. and Sarin, R. "Learning through Reinforcement and Replicator Dynamics", Journal of Economic Theory, 77, 1-14, (1997); Easley D. and Rustichini, A. "Choice without Belief", Econometrica, 67, (1999). There is even a growing experimental literature. For an illuminating survey, see Fudenberg D. and Levine, D, The Theory of Learning in Games, MIT Press, Cambridge, MA, (1998).

[8] Shapley, L. and Shubik, M., "Trade Using One Commodity as a Means of Payment", Journal of Political Economy, 85, 937-968, (1977). 
[9] Mézard,M., Parisi,G., and Virasoro, M. A. Spin Glass Theory and Beyond, World Scientific, Singapore, 1987.

[10] D. Challet, M. Marsili and R. Zecchina, Phys. Rev. Lett 84, 1824 (2000). A more detailed account of this work is given in M. Marsili, D. Challet and R. Zecchina, Physica A 280, 522 (2000).

[11] J. Berg, M. Marsili, A. Rustichini and R. Zecchina in preparation. 